\title{
Factors associated with sexual functioning in women following spinal cord injury
}

\author{
J Harrison ${ }^{1}$, CA Glass ${ }^{2}$, RG Owens ${ }^{3}$ and BM Soni ${ }^{2}$ \\ ${ }^{1}$ University of Liverpool, Department of Clinical Psychology, PO Box 147, Liverpool L69 3 BX; ${ }^{2}$ Southport Regional \\ Spinal Injuries Centre, District General Hospital, Town Lane, Southport, Merseyside PR8 6PN; ${ }^{3}$ Department of \\ Health Studies, University College of North Wales, Bangor, Gwynedd LL57 2DG, UK
}

\begin{abstract}
Research into sexuality following spinal cord injury (SCI) has tended to concentrate on male experiences and the physical capabilities for sexual intercourse. The sexuality of women following SCI has only recently been addressed and studies are limited to small numbers and the use of non-standardised measures. The present investigation utilised standard measures of affective state and body satisfaction together with pre and post-injury questionnaire information of sexual dysfunction, feelings about sex and importance of sexual activity in a group of 85 women with SCI. Sexual dysfunction increased significantly post-injury, whilst feelings about sex and it's importance were unaffected. Sexual dysfunction and the importance of sex were inversely correlated. General and Head satisfaction estimates were not significantly different to control samples, whilst Body Satisfaction was increased for women with disabilities. None of the body satisfaction measures were related to the sexual functioning measure. General dissatisfaction was associated depression. Both anxiety and depression were experienced by the same individuals, and anxiety related to current sexual dysfunction. Qualitative data supported previous findings concerning the effects of social and attitudinal barriers on sexual functioning.
\end{abstract}

Keywords: female sexuality; spinal cord injury; body satisfaction; affective state

\section{Introduction}

Bogle ${ }^{1}$ defined sexuality as the 'integration of physical, emotional, intellectual and social aspects of individual's personality expresses maleness or femaleness'. A definition of sexuality that encompasses more than genital and motor functioning is particularly important for people with disabilities, whose opportunities for physical interactions with the world may be limited. A woman who is not able to use parts of her body or her senses, or who does not look like other women, has as much right to full sexual expression as anybody else. As Cole ${ }^{2}$ notes, however, 'society is still struggling with the negative concept that disabled people are asexual and could not possibly have concerns or problems regarding sexuality in the same way as able-bodied people' (p. 277).

Early research looking at sexuality after spinal cord injury tended to have a physiological emphasis, concentrating for men on erectile function and fertility, and for women on reproduction and pregnancy ${ }^{3,4}$. The sexual functioning of men has been dealt with far more extensively in the literature ${ }^{3,5}$. This may be partly explained by the higher incidence of SCI in men than in women. However, a more significant

Correspondence: CA Glass issue is the view that sexual dysfunction is less traumatic for women: 'women's physical re-adjustment is less problematic because they play a passive role in sexual intercourse' ${ }^{6}$.

The female sexual experience is a complex interplay of psychological and somatic processes ${ }^{7}$. Two basic physiological processes underlying the sexual response cycle are vasocongestion and myotonia, which may be initiated by both reflexogenic and psychogenic mechanisms. A recent study ${ }^{8}$ indicated that complete transection of the cord at T10 or above results in total insensitivity in a resting state, which is located in both the external and internal genitalia. Injuries at lower levels or incomplete lesions give rise to partial insensitivity. It was also reported that during excitation, vaginal lubrication and vasocongestion, in both reflex and psychogenic forms, may still occur at all levels in those with an incomplete spinal injury. In those with a complete lesion above T9, only reflex lubrication and congestion may occur.

It is important to understand the differential effects of various types of disability. A congenital disability is likely to permeate all aspects of sexual development; for example, a lack of privacy and independence in daily living may interfere with the process of acquiring sexual knowledge. An acquired disability is likely to 
have different implications depending on the stage of the life at which it occurs. For example, acquiring a disability early in life may have diffuse effects such as the interruption of gender role and sexual development, whereas a woman who acquires a disability after reaching adulthood may be more likely to recognise specific losses. There are also differences between progressive and stable conditions, eg, the uncertainty and anxiety created by a progressive disability could compromise spontaneity and forward planning.

Women with disabilities may have concerns regarding sexuality because they differ physically from ablebodied women, or they may have been denied sex education or information; they may have questions about their role as a woman and, in society, about their femininity and self-image. With increased acceptance of sexual variety and information about sexuality, an increased repertoire of sexual behaviours is indicative of a satisfying sexual relationship following injury ${ }^{5,9,10}$. More attention is now paid to the psychosocial and rehabilitative aspects of sexuality, which are regarded as an essential aspect of comprehensive care.

The aim of the present investigation was to further address the neglected area of sexual issues for women with disabilities. It was also felt important to present some data specific to women living in the UK and who are living independently in the community, especially because the majority of information relating to SCI concerns institutionalised males ${ }^{3}$. As the present study constituted an initial enquiry, it included women with congenital and progressive disabilities affecting the spinal cord alongside women with SCI.

\section{Method}

A detailed questionnaire was distributed to all women aged between 16 and 60 who appeared on the patient database at the Regional Spinal Injuries Centre at Southport. Marital status was not used to select subjects because of the very limited information this provides about sexual behaviour ${ }^{3}$. It was also felt to be important not to exclude women who may have chosen alternative or non-heterosexual lifestyles.

The first section of the questionnaire was designed to establish age, relationship status and injury/ disability history. It deliberately used the terms 'relationship' rather than 'marriage', and 'partner' rather than 'husband' or 'boyfriend'. It sought to reveal not only the neurological level of the injury, but also the degree of functional disability.

The second section was intended to elicit information about the frequency of sexual activity; the frequency and nature of sexual dysfunction; and attitudes towards (aspects of) sexual activity. 'Before' and 'now' measures were obtained in each case.

The third section consisted of the Body Satisfaction Scale (BSS) ${ }^{11}$. This scale consists of a list of 16 body parts, with which the individual rates their satisfaction on a scale of 1 to 7 (higher scores indicate greater dissatisfaction). The scale contains two internallyconsistent subscales which relate to parts of the body above the neck ('head') and below it ('body').

The final section measured levels of anxiety and depression using the Hospital Anxiety and Depression Scale (HAD) ${ }^{12}$. This is a self-assessment scale which can reliably detect the presence of depression and anxiety, and the subscales it yields have been shown to be valid measures of the severity of emotional disorder. Higher scores reflect increased psychological disturbance.

\section{Results}

The number of questionnaires sent out was 226. Of these, $85(37.6 \%)$ were answered and returned, 41 $(18.2 \%)$ were returned unanswered and $100(44.2 \%)$ were not returned. The response rate is highest for the youngest age group (16-25yrs; 10 year groupings), and declines as age increases although these differences were not significant. The response rate shows a similar trend when stratified by time since injury, although this is due, at least in part, to the tendency for the most recently injured to be younger.

Of the 67 women with an acquired disability, 43 $(64 \%)$ were in a sexual relationship at the time of the study; of these, $30(70 \%)$ are still with the same partner. Of the 13 who are not, $8(62 \%)$ report that their injury was the major cause of the break-up. Overall, $71 \%$ of women have a current partner, the average length of relationship being just under 13 years. The frequency of sexual activity pre- and postinjury is summarised (see Figure 1); note that the figures for pre-injury activity do not include those with congenital conditions. A very high proportion $(40 \%)$ do not reveal their current level of sexual activity; this level of non-response is maintained for all the questions to do with present feelings about sex and sexual dysfunction. Only about half these women are not in a current relationship.

Although on average spinally injured women report an increase in sexual dysfunction, their feelings about sex, and their consideration of which aspects of sexual activity are important, remain largely unchanged. In particular, increases in dysfunction tend to be slight exacerbations of problems experienced previous to the injury, especially those related to arousal and orgasm. The correlations between 'before' and 'after' are high (usually $P<0.01$ ), and the standard deviations of the increases are small (averaging 1). Attitudes towards sexual intercourse are, on average, virtually unchanged, although there is a little variation. Aspects of love-making other than foreplay and intercourse, such as holding and touching one's partner, show a slight increase in relative importance, although again there is a moderate level of variation.

The responses to the sexual dysfunction question referring to the ability to achieve orgasm are summarised in Table 1. This table was derived by partitioning degrees of dysfunction into the categories 

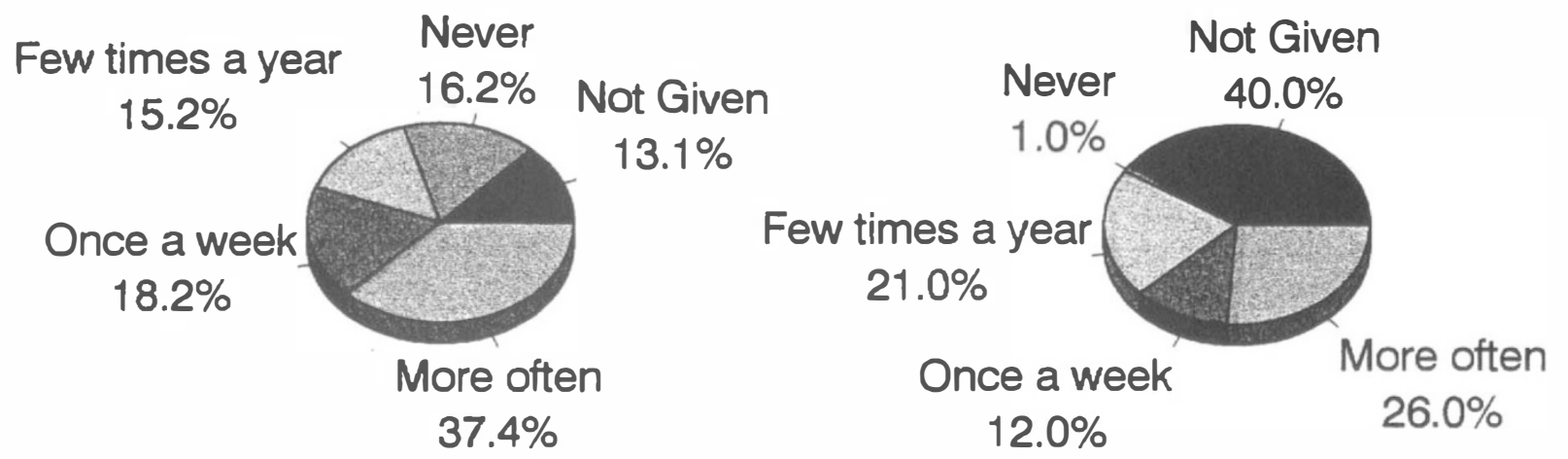

Figure 1 Frequency of sexual activity before injury and at the present time

Table 1 Ability to achieve orgasm before injury and at present time

\begin{tabular}{lcc}
\hline & Before injury & At present time \\
\hline Yes & $39(46 \%)$ & $31(36 \%)$ \\
No & $6(7 \%)$ & $20(23 \%)$ \\
Not applicable & $28(33 \%)$ & $10(12 \%)$ \\
Not supplied & $12(14 \%)$ & $24(28 \%)$ \\
\hline
\end{tabular}

shown. The percentage of women experiencing frequent or persistent inability to achieve orgasm shows a sharp increase, from $7 \%$ to $23 \%$. The percentage of women experiencing little or no problem looks as though it has fallen, but bearing in mind the number of missing answers in each case, about half the women answering can be categorised as 'yes' for both 'before' and 'now'.

Perhaps more reliable are the responses to the question 'Do you think that your spinal problems have affected your sex life?' Of the 71 women who answered, two-thirds indicated that their sex life had been affected for the worse, although about a quarter reported no change. One in 25 felt that their sex life had improved.

In keeping with a broader concept of sexuality, account was taken of how the women feel about their bodies. Satisfaction with the head for women with spinal cord injuries was essentially comparable with control groups of able-bodied women provided by the authors of the $\mathrm{BSS}^{11}$ (see Figure 2). When measuring

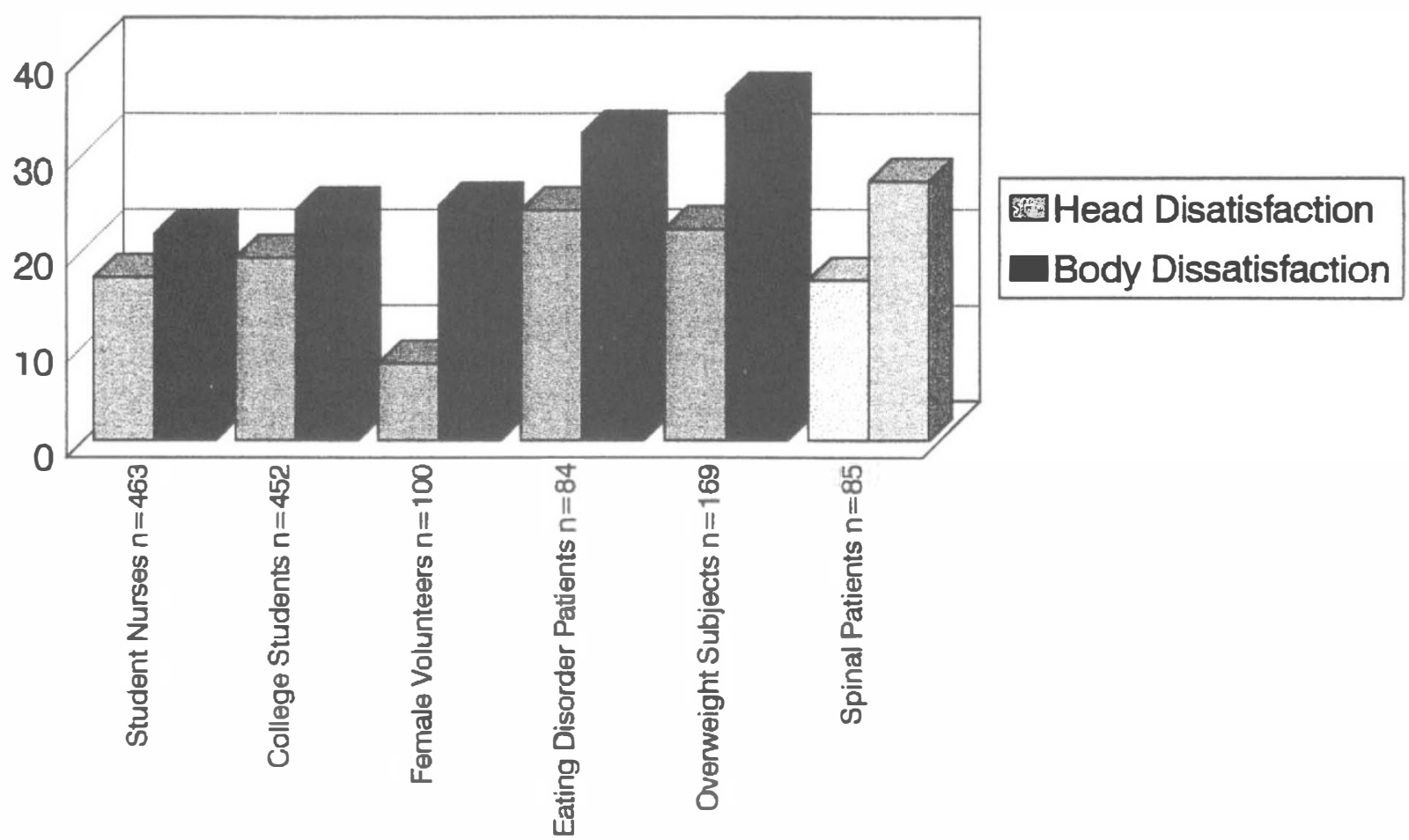

Figure 2 Head and body dissatisfaction scores for spinal patients, compared to a selection of control groups 
Table 2 Distribution of anxiety and depression scores

\begin{tabular}{lcr} 
& Anxiety & Depression \\
\hline Non-cases & $34(43.6 \%)$ & $60(77.9 \%)$ \\
Doubtful & $20(25.6 \%)$ & $8(10.4 \%)$ \\
Cases & $24(30.7 \%)$ & $9(11.4 \%)$ \\
Total & 78 & 77 \\
\hline
\end{tabular}

'body' satisfaction, however, disabled women showed greater dissatisfaction than student nurses, although less than that felt by the eating disorder patients. Generally, trends of dissatisfaction were found to increase with age and the level of disability (but not significantly), although this was not associated with sexual frequency or dysfunction.

The frequency distribution of levels of anxiety and depression is presented in Table 2. Those scoring between 0 and 7 fall into the 'non-case' category; between 8 and 10, 'doubtful'; and between 11 and 21 , 'caseness'. Anxiety and depression are positively correlated $(n=77, r=0.626$, one-tailed $P<0.001)$, and higher scores of anxiety and depression are associated with negative feelings about sexual activity $(r=0.492$, $P<0.001)$. Anxiety is correlated with the frequency of current sexual dysfunction $(r=0.384, P<0.01)$ and depression is associated with general body dissatisfaction $(r=0.455, \mathrm{P}<0.001)$.

\section{Discussion}

There are problems with both questionnaires and interviews, particularly for sexual surveys. Although questionnaires allow a more economical use of the researcher's time, and ensure that questions are standardized, they do not ensure that questions have been understood. There are still limits on the questions that can be asked about sex that are completely without ambiguity and the possibility of misinterpretation, especially if the wording is not to become insensitive or absurd. There is little research evidence to identify which information is best obtained by questionnaire, and which by interview; Although interviews may appear to offer higher face validity for obtaining such information, much may depend on the level of rapport or trust that can be established by the interviewer.

The analyses was performed on the 85 completed questionnaires. This subgroup is, therefore, neither representative of the intended sample hospital population, nor of the female spinally injured population in general. Previous studies using questionnaires in the able-bodied female population have had response rates that are higher, but still relatively low (Gorer, 47\% ${ }^{13}$; Schofield, $51 \%{ }^{14}$ ); the higher refusal rate in this study was to be expected, considering the population under examination. Bancroft ${ }^{7}$ reports that the conditions required for people to answer honestly and adequately are that they see a good reason to reveal the information, and they can trust the recipient to treat that information with respect and confidentiality. By the nature of the study, one would expect the women not to answer the questionnaire, if this was not the case. So, although the information that has been gathered should be regarded as reliable, it is not necessarily representative.

Also to be borne in mind is the significant number of women that did not answer many of the questions relating to their current sexual activity. One might be tempted to believe that these women are not sexually active at the present time, but there is no evidence to support this on the basis of the answers that have been provided.

More than two-thirds of relationships survived the trauma of SCI, although of the 13 that failed, the injury was blamed in 8 cases. The many changes involved in acquiring a disability can place relationships under considerable strain. Relationships with men may be particularly vulnerable when a man has to take on a caring role when the woman's role is often bound up with caring for a male partner ${ }^{15}$. However, the number of sustained relationships goes some way in challenging the myth that people with disabilities do not have partnerships or sexual relationships. In discussing the quality of their relationships, many women stressed the need to be independent of their parents and partners, and particularly the value of open communication (previously underlined by Becker, 1978 ${ }^{9}$ ).

Overall, the women's feelings about sex, and the aspects of sexual activity which they considered important, remained unaffected by their disability. However, there were many causes of the increase in reported sexual dysfunction, ranging from physical and emotional to social. Many of the physical reasons that were reported concerned impaired bowel and/or bladder function, and there were many comments about pain, spasms and cramp. This was not so much an effect on desire, but rather it meant that sexual activity had to be interrupted or stopped. As a result, the expectation of discomfort could also be inhibiting.

It was interesting that current sexual dysfunction was positively correlated with the importance of sex; an increase in problems does not mean a declining interest in sex or involvement in sexual relationships, a conclusion also drawn by Zwerner ${ }^{5}$. It also seems to identify a group of women with specific needs which remain unmet by current services, and underline that sexuality is an important part of overall functioning. The way in which such services may be improved is outlined in a further paper by one of the present authors $^{19}$. It should be remembered, however, that people with disabilities, just as able-bodied people, can have sexual dysfunctions of purely psychological origin, caused by the grief response to loss, or worries about having children or financial difficulties.

The effect that acquired disability can have on sexuality frequently depends on circumstances before the injury ${ }^{15}$. A woman who has pre-injury experience of comfort and delight in her body can often recover 
these good feelings, although the way in which loss is experienced and the kind of initial care given is very important to this process ${ }^{9}$. The view that positive feelings can be regained appears to be partly born out in the present study, in that feelings about sex and important aspects of sexual activity are positively correlated before injury and at the present time. It was felt to he too problematic to ask women to complete the BSS for how they felt before their injury, but longitudinal studies of how body satisfaction may change along the process of adjustment could be useful, although care needs to be taken to prevent confounding due to the effects of increased age on general body dissatisfaction.

In the present study, body satisfaction was not associated with sexual frequency or dysfunction. This could indicate body dissatisfaction / disparagement is not a key feature of 'self-image' which in itself is intrinsic to, and affects, sexual adjustment. Dell Fitting et $a l^{16}$ reported a correlation between 'sense of attractiveness' and being in a sexual relationship. The current results do not show an association between body satisfaction and being in a relationship.

The female spinal patients show an interesting contrast with the student nurses control sample presented in Slade et $a l^{11}$. Just over half the sample were placed in the paraplegia group, most of whom would be using wheelchairs. Paralysis can cause a loss of muscle tone in the stomach and legs; leg muscles may atrophy, and it may be difficult to avoid putting on weight around the stomach region. These effects are likely to account for most of the increased body dissatisfaction in the present sample, evident in the ratings of 'tummy' and 'legs' in the BSS. It could be important, however, to separate out the effects of disability from those of being overweight, as the latter has been shown to have a major influence on body satisfaction ${ }^{11}$. Many women described how part of dealing with a changed body shape was to pay more attention to the head and upper body; these attitudes are likely to have contributed to the head satisfaction scores being consistent with Slade et al ${ }^{11}$ control samples, across all levels of disability.

A comparison of means indicates that female spinal patients are more comfortable with their bodies than the eating disorder and overweight subjects. This may be related to the process of adjustment to a body shape which essentially can not be altered. The correlation between depression and general body dissatisfaction in the spinal patients may reflect a stage in the adjustment process. Longitudinal studies would be needed to investigate such relationships; this gap in the literature is also noted by Willmuth ${ }^{3}$.

The positive correlation between depression and anxiety indicates that an individual may experience both emotional states. In terms of anxiety, a spinal condition may represent the constant stress of coping with disability in an able-bodied world. Access and transport were familiar themes to arise in the interviews. Depression may arise in the response to the many losses; body function, independence, choices. Most interviews revealed that there had been little acknowledgement of the emotional aspects of the women's experience. Sexuality was never mentioned during their care, and they felt this was unhelpful. Neither anxiety nor depression was found to be related to time since injury or degree of disability. It may be that the women in this study are past the acute phase, so that affective state is now related to individual circumstances.

Some internal consistency of the questionnaire was evident in that higher levels of anxiety and depression are associated with negative feelings about sexual activity, since it would be expected that people who are depressed and anxious would also report other negative emotions. In addition, there was an association between anxiety and current sexual dysfunction, and although it is not clear which causes the other, sexual stimulation needs to be accompanied by the appropriate emotional conditions, and anxiety and fear have an inhibiting effect on sexual arousal and response 17,18

The insensitivity to their sexual needs and lack of understanding by health professionals is also regarded by many women as a hindrance to their adjustment to disability in previous reports ${ }^{5,16}$. That most medical professionals are able-bodied and male is likely to have contributed to the historical failure to address issues of sexuality for women with disabilities, together with the cultural taboos associated with sexual expression and the ergonomics of hospitals which frequently preclude notions of privacy and intimacy. The greater numbers of male spinal patients may also have indirectly contributed to some lack of understanding of the needs of women. Many interviewees expressed the opinion that women patients were somehow 'left out' during hospital care, and that there was a degree of sexual stereotyping in what was expected of the male and female patients.

In summary, the experience of women with disabilities on sexuality issues reflect some commonly held stereotypical beliefs and attitudes. This study has examined some variables which are considered to be important in enabling women to begin to redress these matters. The willingness and candour with which women came forward to share their experiences and offer their views suggests that women are unlikely to be inclined to adopt a passive role in the future.

\section{References}

1 Bogle J. A Manual for Service Providers. 1980; Ebon Research Systems, Washington.

2 Cole SS. Women, sexuality and disability. Women and Therapy 1988; 7: $277-294$

3 Willmuth ME. Sexuality after spinal cord injury: a critical review. Clinical Psychology Review 1987; 7: 389-412.

4 Higgins GE. Sexual response in spinal cord injured adults: a review of the literature. Archives of Sexual Behaviour 1979; 8: $173-196$ 
5 Zwerner J. Yes we have troubles but nobody's listening: sexual issues of women with spinal cord injury. Sexuality and Disability 1982; 5(3): $158-171$.

6 Ford AB, Orifier AP. Sexual behaviour and the chronically ill patient. Medical Aspects of Human Sexuality 1967; 10: 51-61.

7 Bancroft J. Human Sexuality and Its Problems 1989. 2nd ed. Churchill-Livingstone, London.

8 Berard EJJ. The sexuality of spinal cord injured women physiology and pathophysiology. Paraplegia 1989; 27: $99-112$.

9 Becker EF. Female Sexuality Following Spinal Cord Injury. 1978; Cheever Publishing Inc., Bloomington, Illinois.

10 Bregman S and Hadley RG. Sexual adjustment and feminine attractiveness among spinal cord injured women. Archives of Physical Medicine and Rehabilitation 1976; 57: 215-227.

11 Slade PD, Dewey ME, Newton T, Brodie D and Kiemle G. Development and preliminary validation of the body satisfaction scale. Psychology and Health 1990; 4: $213-220$
12 Zigmond AS and Snaith RP.The hospital anxiety and depression scale. Acta Psychiatrica Scandinavica 1983; 63: $361-370$.

13 Gorer G. Sex and Marriage in England Today. 1971; Nelson, London.

14 Schofield M. The Sexual Behaviour of Young People. 1965; Longman, London.

15 Morris J. (Ed) Able Lives: Women's Experience of Paralysis. 1989; Women's Press, London.

16 Dell Fitting M, Salisbury S, Davies N and Mayclin DK. Selfconcept and sexuality of spinal cord injured women. Archives of Sexual Behaviour 1978; 7: 143-156.

17 Katchadourian HA. The Biological Aspects of Human Sexuality. 1990; 4th. ed. Holt, Rinehart and Winston, New York.

18 Kitzinger S. Women's Experience of Sex. 1983; GP Puttnam and Sons, New York.

19 Glass CA Addressing psychosexual dysfunction in neurological rehabilitation settings. Journal of Mental Health. 1995; (In Press). 